\title{
Bilateral adrenal hemorrhage in antiphospholipid syndrome
}

\author{
Anticoagulation for the treatment of hemorrhage
}

Hana Aldaajani, MD, Salma Albahrani, MBBS, Khalid Saleh, MD, Khawla Alghanim, MBBS.

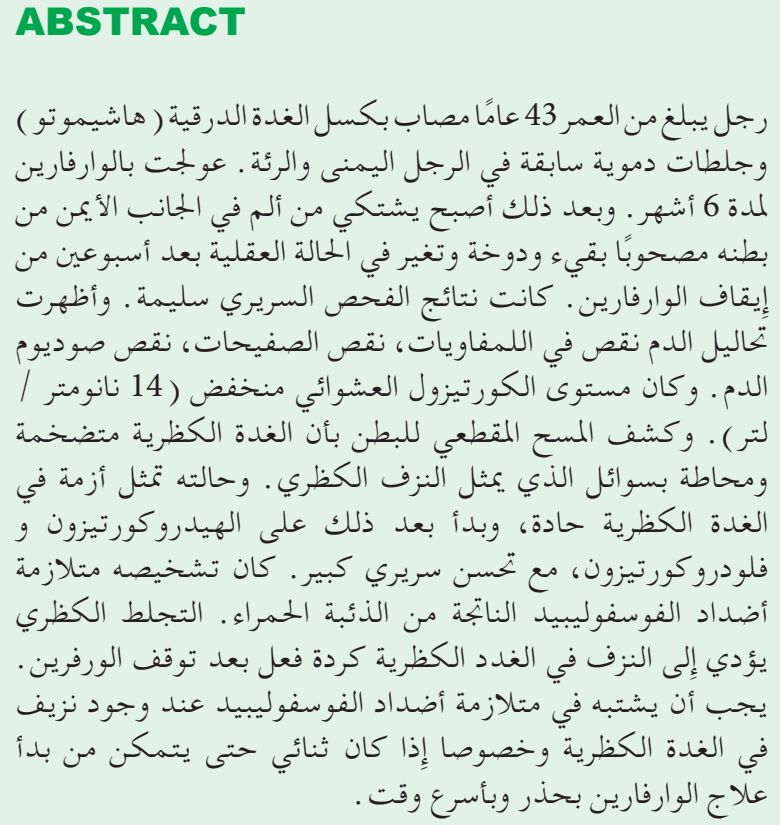

A 43-year-old man with Hashimoto's thyroiditis and previous thromboembolic events treated with warfarin for 6 months, presented with right flank pain accompanied with vomiting, dizziness, and altered mental status 2 weeks after discontinuation of warfarin. His clinical examination findings were unremarkable. Routine blood work showed lymphopenia, thrombocytopenia, and hypoosmolar hyponatremia. Random serum cortisol level was low $(14 \mathrm{nmol} / \mathrm{L})$. Computed tomography scan of the abdomen revealed bilateral bulky heterogeneous suprarenal gland surrounded by fat stranding representing adrenal hemorrhage. He was treated for acute adrenal crisis and subsequently started on hydrocortisone and fludrocortisone, with significant clinical improvement. His diagnosis was secondary antiphospholipid syndrome (APS) to systemic lupus erythematosus (SLE). Bilateral adrenal thrombosis lead to hemorrhage in the adrenals as a paradoxical effect after warfarin cessation and primarily caused by APS. Bilateral adrenal bleeding should lead to the suspicion of thrombophilic disorders, such as APS, with cautious anticoagulation as the treatment of choice.

Saudi Med J 2018; Vol. 39 (8): 829-833

doi: 10.15537/smj.2018.8.22437

From the Department of Internal Medicine (Aldaajani, Albahrani), the Department of Radiology (Saleh), and the Department of Rheumatology (Alghanim), King Fahad Military Medical Complex, Dhahran, Kingdom of Saudi Arabia.

Received 6th March 2018. Accepted 6th June 2018.

Address correspondence and reprint request to: Dr. Khawla Alghanim, Department of Rheumatology, King Fahad Military Medical Complex, Dhahran, Kingdom of Saudi Arabia. E-mail: dr.khawla25@yahoo.com ORCID ID: orcid.org/0000-0002-5226-3254

ntiphospholipid antibodies (Abs) can be seen in Aautoimmune thyroid diseases, hypopituitarism, diabetes mellitus, and ovarian and testicular diseases. Antiphospholipid antibodies may cause vascular thrombi in antiphospholipid syndrome (APS). One of a very rare complication of APS is a primary adrenal insufficiency (AI) resulting from adrenals venous thrombosis, followed by hemorrhagic infarction. The adrenal glands are the most commonly involved glands in APS. ${ }^{1}$ Adrenal insufficiency has been reported in $0.4 \%$ of APS cases and might be the first manifestation of APS. This complication should be identified because it may lead to serious outcomes if left untreated. ${ }^{2}$ Herein, we describe a case of acute AI in a patient with antiphospholipid syndrome (APS) whose adrenal hemorrhage was a paradoxical effect after discontinuation of anticoagulation therapy. Here we describe a similar case of AI due to bilateral adrenal gland hemorrhage after discontinuation of warfarin in a male patient with a previous history of thromboembolic events. His laboratory is very suggestive of APS secondary to SLE. We reviewed the literature of the 
previous clinical cases with similar presentation in APS and we provided a possible underling mechanism that resulted in adrenal necrosis as the exact pathogenesis of adrenal infarction is not yet known in APS. We also provided the appropriate management in such condition as there is no firm guideline in the literature.

Case Report. Patient's information. Our patient was a 43-year-old male with Hashimoto's thyroiditis, right leg deep-vein thrombosis, right lower segmental and sub-segmental pulmonary embolism (PE) treated with warfarin for 6 months, who had no known significant family history of medical diseases. Presented to the emergency room (ER) with right flank pain, nausea, and vomiting for 5 days, along with dizziness, blurring of vision, and disorientation. In the ER, he was observed by the surgical team. All diagnostic exams were inconclusive of acute (surgical) abdominal disease.

Clinical findings. He was found to be confused and hypertensive (blood pressure, 164/94 mmHg). Abdominal examination revealed generalized distention and tenderness.

Diagnostic assessment. Laboratory tests showed lymphopenia, thrombocytopenia, and hypoosmolar hyponatremia (Table 1). Three percent normal saline was administered over 4 hours, and brain CT revealed normal findings.

He was admitted to the medical ward and administered painkillers. Several hours later, he still complained of persistent abdominal pain localized to the right loin. Computed tomography angiography was carried out, which revealed patent arteries and veins with bulky adrenal glands, leading to the possibility of adrenal hemorrhage (Figure 1).

Therapeutic intervention. On the next day, he became hypotensive $(89 / 45 \mathrm{mmHg})$. Intravenous hydrocortisone $100 \mathrm{mg}, 3$ times a day, was initiated for a suspicion of AI, which was then successfully treated. His blood pressure (BP) responded well to hydrocortisone, and he was maintained on oral fludrocortisone and hydrocortisone. Early morning serum cortisol level was low at $14 \mathrm{nmol} / \mathrm{L}$, and 30 minutes post short synacthen test was $320 \mathrm{nmol} / \mathrm{L}$, which supported the diagnosis of AI.

Antiphospholipid syndrome (APS) was suspected

Disclosure. Authors have no conflict of interests, and the work was not supported or funded by any drug company.
Table 1 - Laboratory work upon diagnosis of antiphospholipid syndrome and 2 months post-treatment.

\begin{tabular}{|c|c|c|c|}
\hline Parameters & $\begin{array}{c}\text { On } \\
\text { diagnosis }\end{array}$ & $\begin{array}{c}\text { Two months } \\
\text { post-treatment }\end{array}$ & $\begin{array}{c}\text { Reference } \\
\text { range }\end{array}$ \\
\hline \multicolumn{4}{|l|}{ Hematology } \\
\hline $\operatorname{Hgb}(\mathrm{g} / \mathrm{dl})$ & 13.0 & 15.5 & $13-18$ \\
\hline $\operatorname{WBC}\left(10^{\wedge} 3 / \mu \mathrm{l}\right)$ & 5.09 & 9.3 & $4-11$ \\
\hline Lymphocytes $\left(10^{\wedge} 3 / \mu \mathrm{l}\right)$ & 0.402 & 1.99 & $1.5-4$ \\
\hline Platelets $\left(10^{\wedge} 3 / \mu \mathrm{l}\right)$ & 112 & 249 & $150-450$ \\
\hline PT INR & 1.0 & 2.3 & $0.8-1.1$ \\
\hline PT (seconds) & 12.8 & 29.4 & $10.9-13.6$ \\
\hline APTT (seconds) & 46.8 & 55.7 & $27-38$ \\
\hline TT (seconds) & 15.2 & & $16.7-22.1$ \\
\hline Anti-thrombin III (\%d.N) & 81.4 & - & $74-122$ \\
\hline Fibrinogen $(\mathrm{g} / \mathrm{L})$ & 5.6 & - & $1.7-4.5$ \\
\hline Protein C CH (\%d.N) & 87.9 & - & $60-135$ \\
\hline Protein S AC (\%d.N) & 59.6 & - & $57-126$ \\
\hline \multicolumn{4}{|l|}{ Chemistry } \\
\hline Creatinine $(\mu \mathrm{mol} / \mathrm{L})$ & 137 & 94.0 & $71-115$ \\
\hline BUN (mmol/L) & 4.5 & 10.1 & $2.5-6.4$ \\
\hline $\mathrm{Na}(\mathrm{mmol} / \mathrm{L})$ & 123 & 137.0 & $136-145$ \\
\hline $\mathrm{K}(\mathrm{mmol} / \mathrm{L})$ & 3.0 & 4.3 & $3.5-5.1$ \\
\hline Serum osmolarity (mosm $/ \mathrm{kg}$ ) & 246 & 279.7 & $280-300$ \\
\hline TSH (mIU/L) & 18.1 & 0.78 & $0.52-4.89$ \\
\hline T4 (pmo/L) & 9.42 & 16.2 & $9.92-18.6$ \\
\hline T3 (pmo/L) & 1.71 & 4.2 & $4.36-6.85$ \\
\hline Albumin $(\mathrm{g} / \mathrm{L})$ & 29 & 36 & $34-50$ \\
\hline \multicolumn{4}{|l|}{ Serology } \\
\hline ANA (homogeneous) & $1: 320$ & - & Negative \\
\hline Anti-ds-DNA & $1: 160$ & - & Negative \\
\hline Lupus anticoagulant $\mathrm{Ab}$ & Positive & - & Negative \\
\hline Anti-cardiolipin Abs & Positive & - & Negative \\
\hline Anti-b2-glycoproteinI Ab & Positive & - & Negative \\
\hline $\mathrm{C} 3(\mathrm{~g} / \mathrm{L})$ & 0.49 & 0.9 & $0.9-1.8$ \\
\hline C4 (g/L) & 0.093 & 0.2 & $0.1-0.4$ \\
\hline CRP & 96 & Negative & $<6$ \\
\hline ESR & 62 & 20 & $<15$ (male) \\
\hline \multicolumn{4}{|l|}{ Urine } \\
\hline Microalbumin (mg/L) & 40.4 & - & - \\
\hline 24-h urine protein ( $\mathrm{g} / 24$ hours) & 0.71 & - & - \\
\hline $\mathrm{UACR}(\mathrm{mg} / \mathrm{mmol})$ & 7.06 & $\begin{array}{r}0.130 \mathrm{~g} / 24 \\
\text { hours }\end{array}$ & $0.01-0.16$ \\
\hline
\end{tabular}

Hgb - hemoglobin, WBC - white blood cell, PT/INR - prothrombin time and international normalized ratio, APTT - partial thromboplastin time, TT - thrombin time, Protein $\mathrm{C} \mathrm{CH}$ - protein $\mathrm{C}$ anticoagulant, Protein S AC - protein S activity, BUN - blood urea nitrogen,

$\mathrm{Na}$ - sodium, K - potassium, TSH - thyroid-stimulating hormone, T4 thyroxine, T3 - triiodothyronine, ANA - antinuclear antibody, C3 - complement component 3, C4 - complement component 4, CRP - C-reactive protein, ESR - erythrocyte sedimentation rate, UACR - urine albumin/creatinine ratio

in this patient based on the previous history of thromboembolism and Hashimoto's thyroiditis. A full autoimmune work-up confirmed the presence of anticardiolipin, lupus anticoagulant (LA), and anti-beta-2 glycoprotein I antibodies (Table 1 ). The patient was started on aspirin $81 \mathrm{mg}$ once daily and was kept off anticoagulation for one week. He still experienced bouts of right flank pain. Repeated adrenal CT revealed an interval increase in the size of the adrenal glands, with the right adrenal measuring $33 \times 18 \mathrm{~mm}$, 
and an increase in the attenuation (Figure 2).

Bilateral adrenal hemorrhage was interpreted as a secondary hemorrhage following thrombosis in the venous system of the adrenal cortex within the extent of the APS. Resumption of anticoagulation therapy was decided by the multidisciplinary team to keep the patient's condition stable. The patient was started on unfractionated heparin protocol and warfarin at a dose of $2 \mathrm{mg}$ po OD, with target international normalized ratio (INR) of 2-3.

A follow-up adrenal CT was performed 5 days after

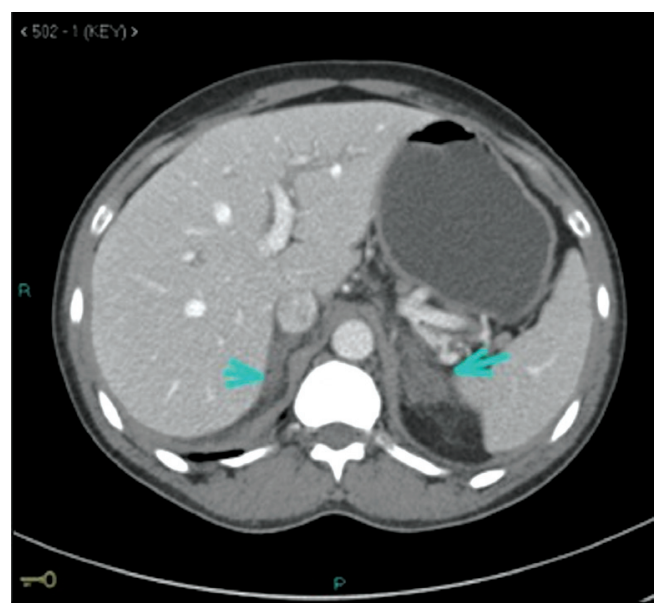

Figure 1 - Selected single axial cut from abdominal computed tomography examination with direct intravenous contrast administration displays that both adrenal glands are enlarged, with reduction of their contrast enhancement, together with surrounding retroperitoneal fat stranding; however, they both show preserved configuration. resumption of anticoagulation therapy, and it showed a reduction in the attenuation from 71 to 55 Hounsfield units (HU) (Figure 3).

Further blood tests revealed the coexistence of systemic lupus erythematosus (lymphopenia, positive antinuclear antibodies, and low complement level) (Table 1) as well as the presence of pericardial effusion in his previous chest CT carried out during his presentation with PE.

The increase in his kidney function tests, presence of blood and proteins in the urine (Table 1), and high BP readings were indicative of proliferative lupus nephritis. A renal biopsy was deferred because the risk of bleeding was very high in the presence of adrenal hemorrhage. He was started on hydroxychloroquine $300 \mathrm{mg}$ po OD, and mycophenolate mofetil $1000 \mathrm{mg}$ po BID for proliferative lupus nephritis. He was discharged home in good condition, with significant improvement in his clinical condition and blood work-up results, but required close follow-up at a rheumatology clinic (Table 1).

Follow up and outcomes. Six months after the initial event, the patient continued to register undetectable levels of plasma cortisol. As part of the follow-up, adrenal CT was performed 7 months later, which showed attenuation in size of both adrenal glands with fibro-fatty replacement and disappearance of fat stranding (Figure 4). One year later, he remains on hydrocortisone and fludrocortisone replacement and continues to have normal serum sodium, potassium, creatinine, and urine analysis. A summary of the patient's history and timeline of the outcomes is given in (Table 2).

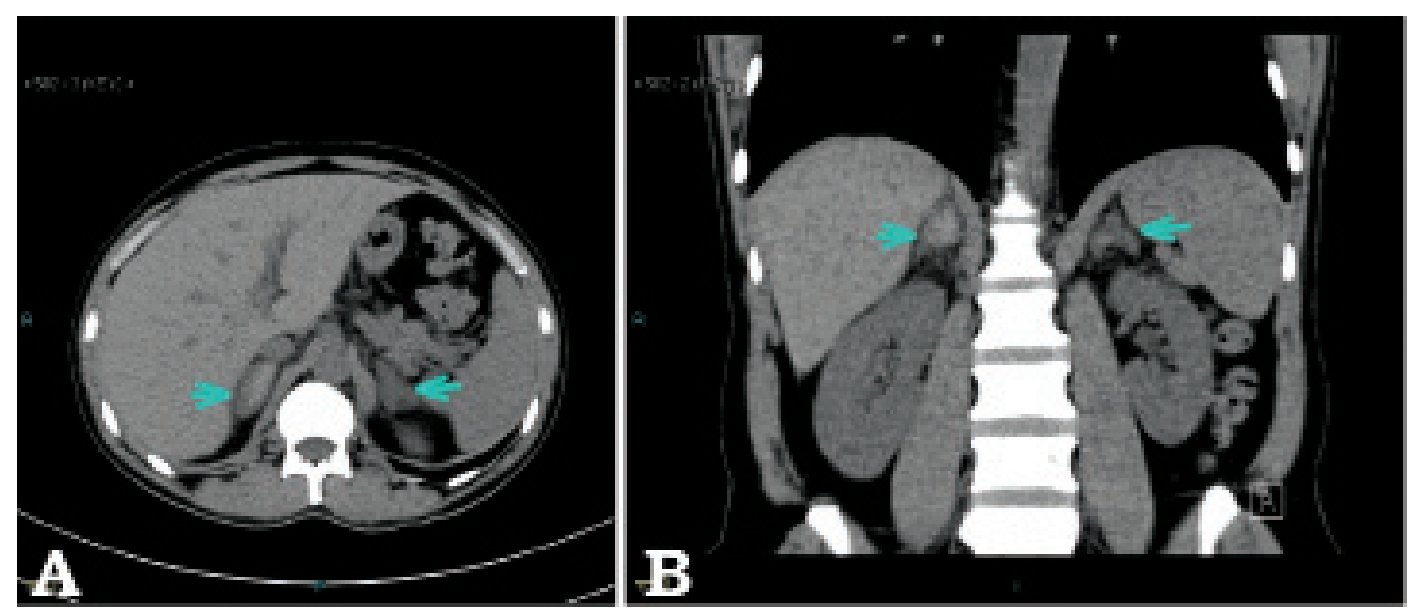

Figure 2 - Non-contrast abdominal computed tomography. A) Selected single axial cut demonstrates more progressive enlargement of the adrenal glands, during interim, with increased attenuation of their central part, about 71 Hounsfield units, and increasing surrounding fat stranding, consistent with bilateral adrenal hemorrhage. B) Selected coronal cut confirms the findings seen on Figure $2 \mathrm{a}$ and displays the characteristic triangular configuration of adrenal glands. 

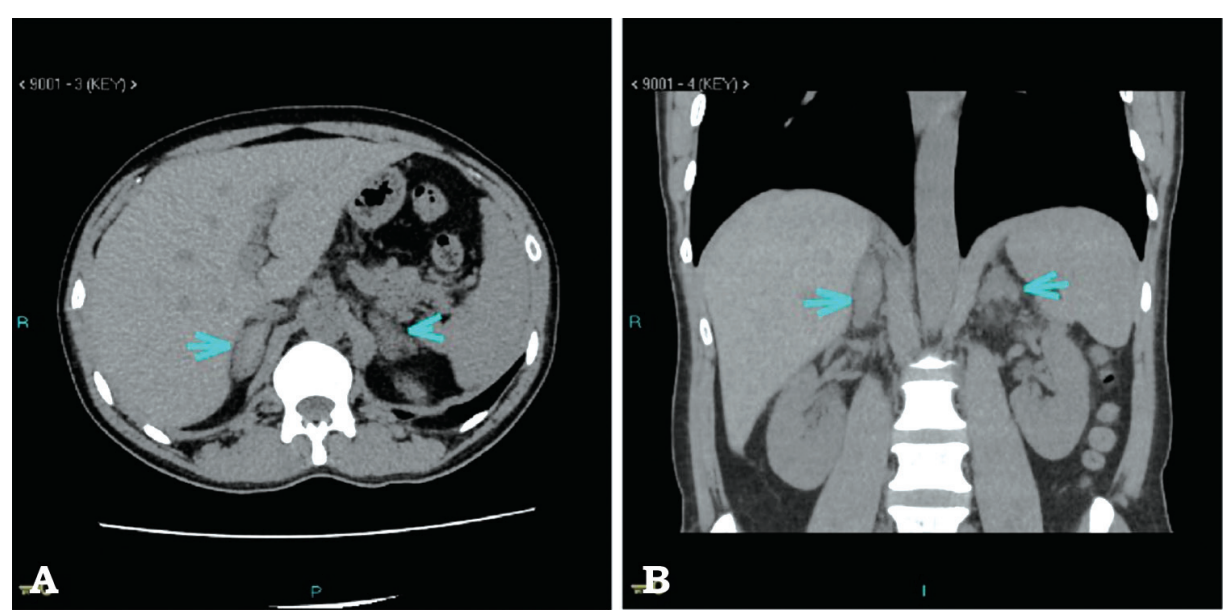

Figure 3 - Non-contrast abdominal computed tomography. A) Selected single axial cut shows that the size of the adrenal glands is still enlarged i.e. does not change since the previous examination; however, the attenuation values are slightly reduced approximately from 71 to $55 \mathrm{HU}$ in the current examination. Surrounding fat stranding is still seen. B) Selected single coronal cut confirms the abnormalities seen on figure $3 a$.
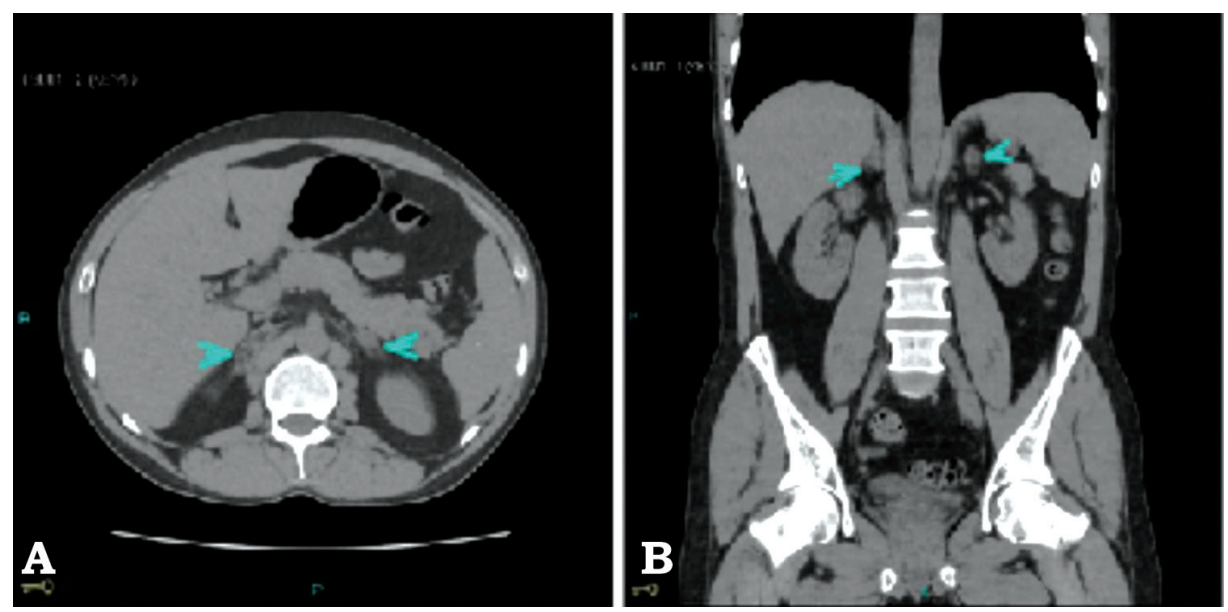

Figure 4 - A) Non-contrast abdominal computed tomography 7 months later. Both adrenal glands displayed size and attenuation value reduction, with fibro-fatty replacement, and the retroperitoneal fat stranding disappeared. The attenuation value was approximately $20 \mathrm{HU}$. No calcifications were observed. B) Selected single coronal cut confirms the abnormalities seen on figure 4a.

Discussion. Antiphospholipid syndrome is a prothrombotic autoimmune disorder characterized by medium-to-large vessel thrombosis or pregnancy complications. As APS can affect veins and arteries, as well as virtually any organ in the body, it has various clinical features. Adrenal insufficiency is a rare complication of APS; however, it may also be the first clinical presentation of the syndrome. In the present case, the clinical presentation of AI is fairly typical of the reported cases of hemorrhagic infarction of the adrenals due to APS, with symptomatic hyponatremia and abdominal pain due to AI. Abdominal pain shown to be the most common symptom, occurring in $55 \%$ of cases. Other presentations include hypotension $(54 \%)$, fever $(40 \%)$, nausea or vomiting (31\%), weakness/fatigue (31\%), and altered mental state (19\%). Nonetheless, it is important to have a high level of clinical suspicion of AI in any patient with a history of APS who presents with abdominal pain. In addition to the blood tests, such as serum sodium, serum potassium, random cortisol and short Synacthen test, imaging techniques, such as abdominal CT or MRI, should also be undertaken. This case illustrates the importance of promptly recognizing AI in patients with APS and the possible causative agents, which require careful consideration and exclusion to prevent 
Table 2 - Summary of the patient case from initial visit to treatment and follow -up.

\begin{tabular}{|c|c|}
\hline Date & History/intervention/outcomes \\
\hline \multicolumn{2}{|l|}{ Year 2017} \\
\hline 9th February & Patient presented to ER with abdominal pain \\
\hline 10th February & $\begin{array}{c}\text { CT angiography abdomen ( bilateral adrenal glands } \\
\text { bleeding) }\end{array}$ \\
\hline 11th February & $\begin{array}{l}\text { Patient became hypotensive, early morning serum } \\
\text { cortisol level and } 30 \text { minutes post short synacthen } \\
\text { test supported (AI), IV hydrocortisone and } \\
\text { fludrocortisone, CT brain (normal). }\end{array}$ \\
\hline 12th February & Serology tests for APS and SLE, Aspirin Started \\
\hline 15th February & $\begin{array}{l}\text { Repeated adrenal CT ( bleeding increased), } \\
\text { resumption of Warfarin }\end{array}$ \\
\hline 20th February & $\begin{array}{l}\text { Repeated adrenal CT (bleeding decreased), serology } \\
\text { tests supported APS and SLE }\end{array}$ \\
\hline 23 th February & $\begin{array}{l}\text { High dose prednisolone, hydroxychloroquine and } \\
\text { MMF were started for SLE, discharge home with } \\
\text { close follow up at rheumatology clinic (every month). }\end{array}$ \\
\hline 4th August & $\begin{array}{l}\text { Follow up at rheumatology clinic, normal blood } \\
\text { work-up results, urine analysis and } 24 \text { urine protein, } \\
\text { plasma cortisol level was undetectable }\end{array}$ \\
\hline 3rd September & Repeated adrenal CT (bleeding disappeared) \\
\hline \multicolumn{2}{|l|}{ Year 2018} \\
\hline 28th February & $\begin{array}{l}\text { Follow up at rheumatology clinic, normal blood } \\
\text { work-up results, urine analysis and } 24 \text { urine protein }\end{array}$ \\
\hline 30th February & $\begin{array}{l}\text { Follow up at endocrine clinic, continue } \\
\text { hydrocortisone and fludrocortisone for } \mathrm{AI}\end{array}$ \\
\hline \multicolumn{2}{|c|}{$\begin{array}{c}\text { ER - emergency room, CT - computed tomography, AI - adrenal } \\
\text { insufficiency, IV - intravenous, APS - autoimmune polyglandular } \\
\text { syndrome, SLE - systemic lupus erythematosus, MMF - mycophenolate } \\
\text { mofetil }\end{array}$} \\
\hline
\end{tabular}

further thrombotic events. Furthermore, it highlights the importance of imaging techniques, such as $\mathrm{CT}$, in patients with APS presenting with abdominal pain as well as concomitant autoimmune conditions. When considering the underlying mechanism that resulted in adrenal necrosis, multiple causes could trigger our patient's presentation. While the exact pathogenesis of adrenal infarction is not known in APS, it has been hypothesized that the hypercoagulable state leads to adrenal vein thrombosis, which results in hemorrhagic infarction of the adrenal gland. The infarctions are often bilateral and thought to localize to this area partly due to the unique and peculiar anatomical structure of the adrenal vasculature. The adrenal gland comprises a rich arterial supply with a single vein limiting blood drainage, which when thrombosed can result in progressive increase in arterial blood pressure. There is a predisposition to intraglandular hemorrhage because of an intrinsically vulnerable network; this results from a "vascular dam" that is created from the sharp transition of the artery to the capillary system around the zona reticularis. In certain circumstances, adrenal infarction can be due to local stasis of blood rather than thrombosis.
This in turn increases arterial blood flow that exceeds the limited venous drainage. ${ }^{3}$ Imaging modalities used to visualize adrenal hemorrhage include $\mathrm{CT}$ and magnetic resonance imaging. Acute hemorrhage can appear as a hyper-dense mass on CT scan ${ }^{5}$, and cause enlargement of one or both adrenal glands. ${ }^{4,5}$ A hematoma appears on CT images as a circular non-enhancing mass of greater than simple fluid attenuation (namely, 50-90 HU). ${ }^{6,7}$ In the present case, the cessation of anticoagulation therapy paradoxically led to hemorrhage. Patients with APS should be immediately treated with anticoagulation to avoid further complications. Antiphospholipid syndrome can be fatal if it affects different organs in the body. In cases of APS involving bilateral adrenal gland hemorrhages, careful anticoagulation therapy with unfractionated heparin and warfarin, with a target INR of 2-3, is the treatment of choice to prevent further thrombosis. ${ }^{4}$ Our patient was informed about his clinical condition and agreed with multidisciplinary team to resume his previous anticoagulation with close follow up to his adrenal glands. He was discharged home on warfarin, hydroxychloroquine, mycophenolate mofetil, prednisolone and fludrocortisone with significant improvement in his clinical condition and blood work-up results. One year later, he continues to have stable condition and normal blood work-up results.

In conclusion, bilateral adrenal bleeding should lead to the suspicion of APS as a cause of thromboembolic disorder as this might lead to fatal complications if left untreated. Cautious anticoagulation with warfarin is the treatment of choice with target INR of 2-3.

Acknowledgment. We would like to thank editagae.com for English Editing of our paper.

\section{References}

1. Uthman I, Salti I, Khamashta M. Endocrinologic manifestations of the antiphospholipid syndrome. Lupus. 2006; 15: 485-489.

2. Calvo Romero JM, Morales Pérez F, Alvarez Barreiro JA, Díaz Pérez de Madrid J, Pérez Miranda M. [Primary adrenal insufficiency and primary antiphospholipid syndrome]. An Med Interna 2000; 17: 491-493. Spanish

3. Batt NM, Malik D, Harvie M, Sheth H. Non-haemorrhagic, bilateral adrenal infarction in a patient with antiphospholipid syndrome along with lupus myocarditis. BMJ Case Rep 2016; 2016: pii: bcr2016216364.

4. Anastasia M. Canacci, Gregory T. MacLennan. Adrenal hemorrhage. J Urol 2007; 128: 284.

5. Provenzale JM, Ortel TL, Nelson RC. Adrenal hemorrhage in patients with primary antiphospholipid syndrome: imaging findings. AJR Am J Roentgenol. 1995; 165: 361-364.

6. Gowda D, Shenoy V, Malabu U, Cameron D, Sangla K. Bilateral adrenal gland haemorrhage: an unusual cause. Endocrinol Diabetes Metab Case Rep. 2014; 2014: 140058.

7. Jordan E, Poder L, Courtier J, Sai V, Jung A, Coakley FV. Imaging of nontraumatic adrenal hemorrhage. AJR Am J Roentgenol 2012; 199: W91-98. 